\section{P281 THE EFFECTIVENESS OF ACUPUNCTURE IN MANAGING SYMPTOMS IN CF ADULTS}

${ }^{1}$ EF Nash, ${ }^{2} \mathrm{H}$ Bradley, 'E Chapman, ${ }^{1} \mathrm{R}$ Rashid, ${ }^{1} \mathrm{JL}$ Whitehouse. 'West Midlands Adult Cystic Fibrosis Centre, Heart of England NHS Foundation Trust, Birmingham, UK; ${ }^{2}$ British Acupuncture Council, London, UK

\subsection{6/thoraxjnl-2015-207770.417}

Introduction and objectives People with cystic fibrosis (CF) experience a wide spectrum of symptoms, both physical and psychological, which are often troublesome and can result in significantly impaired quality of life. Acupuncture has a strong evidence base in the treatment of a wide variety of symptoms, however there is currently very limited evidence for its role in the management of symptoms in people with CF. The objective of this pilot study was to provide preliminary evidence of the effectiveness and patient acceptance of this treatment in our large regional adult CF centre.

Methods In this observational prospective study, we offered acupuncture to $\mathrm{CF}$ inpatients attending our large regional adult $\mathrm{CF}$ centre if they were suffering from range of symptoms. Patients were asked to specify the symptoms being treated and its severity before and after treatment, as well as whether treatment achieved the desired outcome, whether they suffered any ill effects, whether it is a valuable service and whether they feel we should continue to offer acupuncture to patients. They were also offered the option of making free-text comments on their experience of the service.

Results 106 patients were included over a 12-month period. 50 patients were treated for pain (back/neck/shoulder pain, $\mathrm{n}=28$; unspecified location, $\mathrm{n}=12$; headache, $\mathrm{n}=5$, chest pain, $\mathrm{n}=$ 4 , toe pain, $\mathrm{n}=1$ ) with pain significantly reducing after treatment (median severity 7 (IQR 6-8) vs. 4 (IQR $3-5$ ), p $<0.001$ ). 25 patients were treated for stress/anxiety with symptoms significantly reducing after treatment (median severity 8 (IQR 7-9) vs. 5 (IQR 3.6-6), p < 0.001). 10 patients were treated for breathlessness/tight chest with symptoms significantly reducing after treatment (median severity 7 (IQR 6-8) vs. 5 (IQR 4-6), p = 0.001). 21 patients were treated for a range of other symptoms including low energy levels, reduced appetite and constipation. 95 patients reported that treatment had achieved the desired result and 10 patients reported that it was too early to tell. 1 patient felt 'a bit sick' after treatment, but no other adverse effects were reported. All patients felt that acupuncture was a valuable service and should continue to be offered. 30 patients commented that the service should be available more frequently. Conclusions Acupuncture was greatly appreciated by CF adults, with significant improvements in a wide variety of symptoms. A randomised controlled study is required to confirm these benefits.

\section{Investigating lung disease: novel techniques and old interventions}

\section{P282 THE DESIGN AND VALIDATION OF A NOVEL SEMIAUTOMATIC LUNG NAVIGATION PLATFORM}

${ }^{1} \mathrm{KA}$ Khan, ${ }^{2} \mathrm{P}$ Nardelli, ${ }^{2} \mathrm{~J}$ Alex, ${ }^{2} \mathrm{C}$ O'Shea, ${ }^{2} \mathrm{P}$ Cantillon-Murphy, ${ }^{1} \mathrm{MP}$ Kennedy. ${ }^{1}$ Department of Respiratory Medicine, Cork University Hospital/University College Cork, Cork, Ireland; '2School of Engineering, University College Cork, Cork, Ireland

10.1136/thoraxjnl-2015-207770.418
Introduction In the era of lung cancer screening, tissue acquisition of peripheral lung lesions remains a challenge. We have developed a $3 \mathrm{D}$ electromagnetic navigation platform with airway segmentation and virtual bronchoscopy using a open source 3D slicer environment.

Methods The open source visualisation software (3D Slicer www.slicer.org) created a detailed airway segmentation and virtual bronchoscopy model from acquired CT images. A magnetic field emitter board provides tracking of a semiautomatic locatable sensor probe (SALP) in the working channel of the bronchoscopewith always-on tip tracked sensor and can be steered both manually and automaticaly with joy stick, for accurate localization of peripheral lung lesion.

An extensive ex-vivo evaluation was performed in a breathing lung model that was developed using inflatable plasticized pig lungs in a negative-pressure Following this, in-vivo real time navigation in a live porcine model using a selection of novel radioopaque fiducials placed endobronchially into distal airways.

Results After completion of a selection of experiments using the breathing pig lung model, fiducials were placed endobronchially in our live porcine model. Thereafter, CT images were used to create a virtual airway $3 \mathrm{D}$ segmentation model. After multiplaner re-construction, land mark based registration was performed to align the CT and anaesthetised porcine. Manual and automatic navigation with the bronchoscope containing the SALP was performed. The average navigation distance covered was $85.3 \mathrm{~mm}$. The navigational system accurately determined $84 \%$ of the navigation points within the airways.

Conclusion Our navigational platform is inexpensive and open source and is the first to utilise SALP. In our model, there is good agreement between the position of the sensor probe during bronchoscopic navigation and as visualised in virtual bronchoscopy. Further work is being carried out to improve registration and accuracy of the navigational system before a pilot study in patients with peripheral lung nodules.

\section{P283 HYPERPOLARISED GAS MRI - A PATHWAY TO CLINICAL DIAGNOSTIC IMAGING}

${ }^{1} \mathrm{JM}$ Wild, ${ }^{1} \mathrm{G}$ Collier, ${ }^{1} \mathrm{H}$ Marshall, ${ }^{1} \mathrm{~L}$ Smith, ${ }^{1} \mathrm{G}$ Norquay, ${ }^{1} \mathrm{AJ}$ Swift, ${ }^{1} \mathrm{FC}$ Horn, ${ }^{1} \mathrm{~F}$ Chan, ${ }^{1} \mathrm{NJ}$ Stewart, ${ }^{1} \mathrm{LC}$ Hutchison, ${ }^{1} \mathrm{M}$ Rao, ${ }^{1}$ I Sabbroe, ${ }^{2} \mathrm{R}$ Niven, ${ }^{2} \mathrm{~A}$ Horsley, ${ }^{3} \mathrm{~S}$ Siddiqui, ${ }^{1} \mathrm{~K}$ Ugonna, ${ }^{4} \mathrm{R}$ Lawson. 'University of Sheffield, Sheffield, UK; ${ }^{2}$ North Western Lung Clinic, Manchester, UK; ${ }^{3}$ University of Leicester, UK; ${ }^{4}$ Sheffield Teaching Hospitals Trust

\subsection{6/thoraxjnl-2015-207770.419}

Introduction Despite the excellent functional sensitivity of hyperpolarised gas MRI to early lung disease, clinical uptake of the technique has to date been hindered by patents, regulatory classification, availability of ${ }^{3} \mathrm{He}$ and access to polariser technology. However, many of these constraints have been alleviated in recent years, and ${ }^{129} \mathrm{Xe}$ MRI is now providing high quality lung images at relatively low cost. In January 2015 UK regulatory approval for the manufacture of hyperpolarised gases for routine clinical lung imaging was obtained in Sheffield. Here we present a case series as an overview of the clinical questions that this technology can help resolve in various respiratory indications.

Methods More than 20 patients (aged 13 to 74 ) have been clinically referred to date with HP gas MRI in Sheffield to date from NHS hospitals across the UK. Clinical histories include non-CF bronchiectasis (scanned before and after a 2 week course of IV antibiotics), COPD for consideration for LVRS/EB valves, 